\title{
Neutron star structure from a quark-model baryon-baryon interaction
}

\author{
K. Fukukawa, ${ }^{1}$ M. Baldo,${ }^{2}$ G. F. Burgio,${ }^{2}$ and H.-J. Schulze ${ }^{2}$
}

${ }^{1}$ Research Center for Nuclear Physics, Osaka University, 10-1, Mihogaoka, Osaka, 567-0047, Japan

${ }^{2}$ INFN, Sezione di Catania, Via Santa Sofia 64, I-95123, Catania, Italy

\begin{abstract}
We derive the equation of state (EOS) of nuclear matter from a realistic constituent quark model for the nucleon-nucleon interaction. We use the Brueckner-Bethe-Goldstone approach with the inclusion of the three hole-line contribution. We find that the resulting EOS reproduces correctly the saturation point, moreover the symmetry energy at low density, its slope, and the incompressibility turn out to be compatible with phenomenology. We calculate the mass-radius relation for neutron stars, and find maximum values close to two solar masses, in agreement with recent observational data.
\end{abstract}

\section{Introduction}

One of the most fundamental issues in theoretical nuclear physics is the understanding of nuclear many-body systems starting from realistic nucleonnucleon $(N N)$ and three-nucleon $(3 N)$ interactions. In particular, it is essential to devise an elementary interaction among nucleons which is able to describe consistently both few-body systems and nuclear matter properties, such as the experimental binding energy of light nuclei and the correct saturation point.

Quark-Model (QM) baryon-baryon (BB) interactions have been modeled to describe the short-range repulsion with the resonating group method for 
(a)

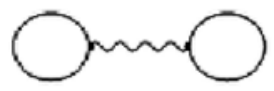

(d)

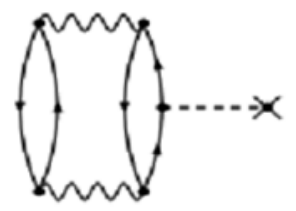

(b)

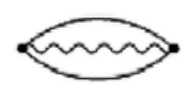

(e)

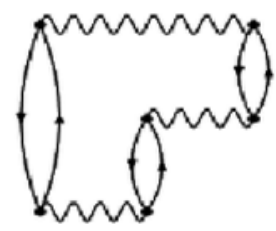

(c)

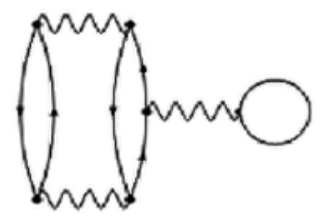

(f)

Figure 1: Different Goldstone diagrams contributing to the nuclear matter EOS. The wavy line indicates the Brueckner $G$-matrix. For details, please see the text.

two $(0 s)^{3}$ three-quark clusters. One of the most developed version of the QM BB interaction, called fss2 [1], has been successfully applied to the threeand four-nucleon systems without resorting to $3 N$ interactions [2]. In this model, the role of $3 N$ interactions may be not so large as usually considered.

In the following we will discuss the EOS of nuclear matter [3] derived with the Bethe-Brueckner-Goldstone (BBG) approach, where the $N N$ interaction is obtained using the fss2. In $\S 2$, we explain the theoretical framework and show our numerical results both for the equation of state and the structure of neutron stars. The final section is devoted to the conclusions.

\section{Nuclear matter EOS and neutron star structure}

We analyze the EOS within the many-body microscopic approach based on the BBG expansion up to three hole-line (THL) level. We show in Fig. 1 the Goldstone diagrams whose contribution has been calculated following Refs. $[4,5]$. Diagrams (a) and (b) correspond to the Brueckner-Hartree-Fock (BHF) calculations, which include the two hole-line contribution, whereas the sum of the other diagrams gives the THL contribution. In Fig. 2 we display for symmetric matter each contribution to the EOS obtained in the so-called continuous choice (CC) for the single-particle (s.p.) potential. For details concerning the theoretical approach, the reader is referred to 


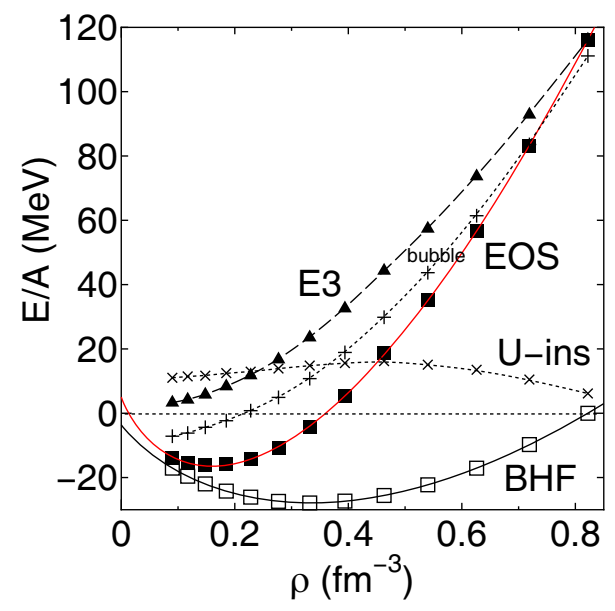

Figure 2: The binding energy as a function of nucleon density is shown for symmetric nuclear matter in the continuous choice. The contribution from the BHF calculation [open squares], the bubble diagram [Fig. 1(c), '+' symbols], the corresponding $U$-insertion diagram [Fig. 1(d), crosses], the total contribution from the THL diagrams [Figs. 1(c)-(f), black triangles], and the resultant EOS [black squares], are plotted separately. The contributions from the ring diagram [Fig. 1(e)], and the higher-order term [Fig. 1(f)] are not shown, because they are small.

Refs. $[3,5]$. The peculiar feature is that THL give large contributions at high densities in the fss 2 case, and this is at variance with the results found in the case of the Argonne $v_{14}$ or $v_{18} N N$ potentials, where the THL are small [6]. We found that the THL contribution is important to predict the correct saturation point $\left[\rho_{0} \approx 0.157 \mathrm{fm}^{-3},(E / A)\left(\rho_{0}\right) \approx-16.3 \mathrm{MeV}\right]$, and an incompressibility $K=219 \mathrm{MeV}$ compatible with phenomenological data. Around saturation density, the contributions from the $U$-insertion diagram are appreciable, whereas at high densities the contributions from the bubble diagram rapidly increase. Since the THL contribution is larger than the BHF contribution at high densities, the BBG expansion becomes questionable beyond a density range where it can be expected that strange baryonic matter and/or quark deconfinement may appear.

In Fig. 3 we show the fss2 EOS for symmetric (SNM) and pure neutron matter $(\mathrm{PNM})$, both compared with other theoretical predictions. In particular, we show results obtained with the well-known APR EOS (dotdashed curve) [7], and with the BHF approach (dotted curve) [8]. In those cases the Argonne $v_{18} N N$ potential is used along with the Urbana model for $3 N$ forces [9]. The other curves show results obtained with BHF and 

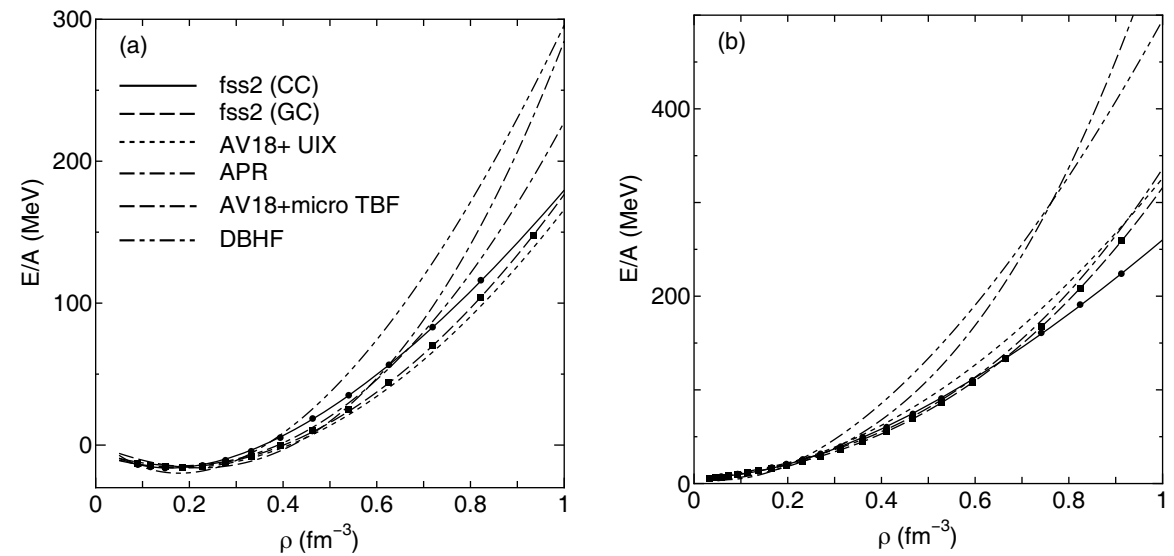

Figure 3: EOS of (a) symmetric nuclear matter and (b) pure neutron matter. The calculations in the continuous and gap choice are denoted by (CC) and (GC), respectively.

microscopic $3 N$ forces [10], and the relativistic DBHF [11]. We observe that the fss2 EOS is quite soft at high densities, and that all EOS agree well in symmetric matter up to nucleonic density $\rho \sim 0.5 \mathrm{fm}^{-3}$. The fss 2 EOS in the continuous and the gap choice agree well with each other up to nucleonic density $\rho \sim 0.7 \mathrm{fm}^{-3}$, so that the final EOS turns out to be independent of the choice of the s.p. potential.

For studying the neutron star structure, we first calculate the composition $\left\{\rho_{i}(\rho)\right\}$ and the EOS for asymmetric, neutrally charged and beta-stable matter. We adopt a conventional description of stellar matter as composed of neutrons, protons, electrons, and muons. The EOS reads

$$
P(\rho)=\rho^{2} \frac{d}{d \rho} \frac{\varepsilon\left(\left\{\rho_{i}(\rho)\right\}\right)}{\rho}=\rho \frac{d \varepsilon}{d \rho}-\varepsilon,
$$

being $\epsilon$ the energy density. For non-rotating and spherically-symmetric neutron stars, the Tolman-Oppenheimer-Volkoff equations [12] must be solved

$$
\begin{aligned}
& \frac{d P}{d r}=-\frac{G m \varepsilon}{r^{2}} \frac{(1+p / \varepsilon)\left(1+4 \pi r^{3} p / m\right)}{1-2 G m / r}, \\
& \frac{d m}{d r}=4 \pi r^{2} \varepsilon,
\end{aligned}
$$




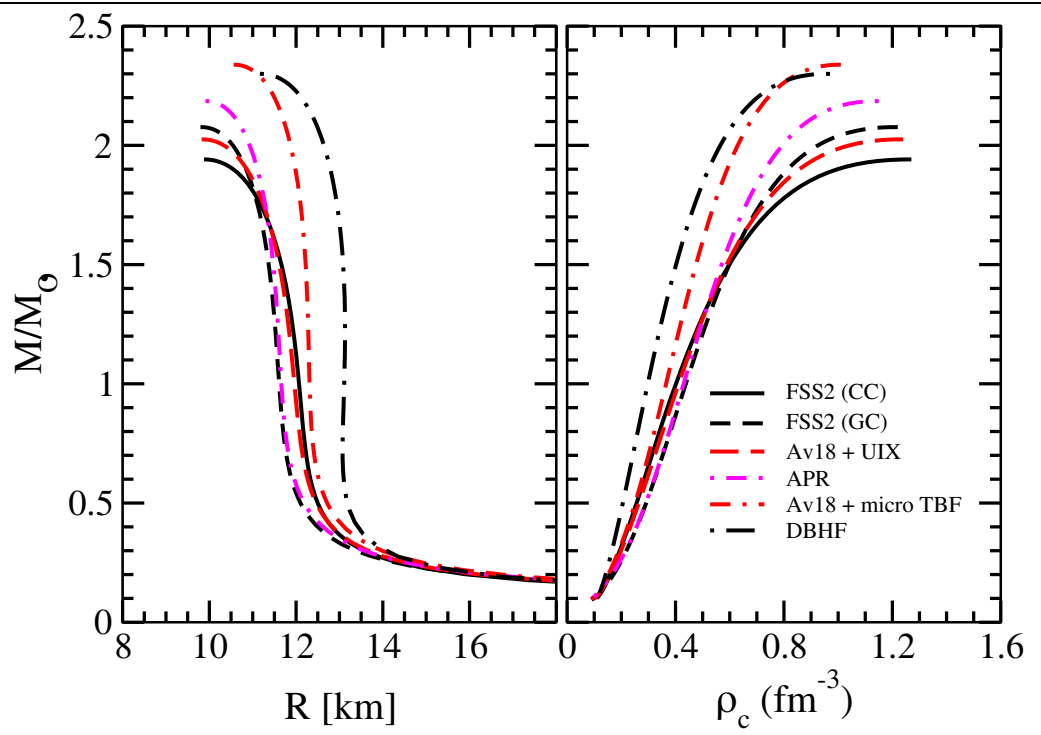

Figure 4: Neutron star mass as a function of radius (left panel) and central baryon density (right panel) for several EOS.

where $G$ is the gravitational constant. We integrate Eqs. (2) from the center to the surface $r=R$ and obtain the gravitational mass $M_{G}=m(R)$. In Fig. 4, we show the mass-radius relation (left panel) and the mass-central density relation (right panel) for the cases discussed above. The fss2 EOS gives slightly different maximum masses for the GC and CC approximations, in line with their different stiffness at high density. However, the range of the maximum mass predicted by fss 2 turns out to be compatible with the largest mass observed up to now, which is $(2.01 \pm 0.04) M_{\odot}[13]$.

\section{Summary}

We have derived the fss2 EOS within the BBG approach up to the THL level of approximation. It provides a saturation point compatible with experimental constraints and reproduces the binding energy of three- and four-nucleon systems without introducing $3 N$ interactions. We found that the $3 N$ correlations are important to predict the saturation point. The obtained neutron star maximum mass is about 2 solar masses. 


\section{Acknowledgement}

The authors wish to acknowledge the "Newcompstar" COST Action MP1304.

\section{References}

[1] Y. Fujiwara et al., Prog. Part. Nucl. Phys. 58, 439 (2007).

[2] Y. Fujiwara, Y. Suzuki, M. Kohno, and K. Miyagawa, Phys. Rev. C 77, 027001 (2008); Y. Fujiwara and K. Fukukawa, Few-Body Systems 54, 2357 (2013); Y. Fujiwara, Few-Body Systems 55, 993 (2014).

[3] M. Baldo, and K. Fukukawa, Phys. Rev. Lett. 113, 242501 (2014); K. Fukukawa et al., arXiv:1507.07288 [nucl-th] (2015).

[4] R. Rajaraman and H. Bethe, Rev. Mod. Phys. 39, 745 (1967); B. D. Day, Phys. Rev. C 24, 1203 (1981), Phys. Rev. Lett. 47, 226 (1981).

[5] M. Baldo, in Nuclear Methods and the Nuclear Equation of State, International Review of Nuclear Physics Vol. 8, pp. 1 (World Scientific, 1999).

[6] H. Q. Song, M. Baldo, G. Giansiracusa, and U. Lombardo, Phys. Rev. Lett. 81, 1584 (1998).

[7] A. Akmal, V. R. Pandharipande, and D. G. Ravenhall, Phys. Rev. C 58, 1804 (1998).

[8] G. Taranto, M. Baldo, and G. F. Burgio, Phys. Rev. C 87, 045803 (2013).

[9] J. Carlson, V. R. Pandharipande, and R. B. Wiringa, Nucl. Phys. A401, 59 (1983).

[10] W. Zuo, A. Lejeune, U. Lombardo, and J.-F. Mathiot, Nucl. Phys. A706, 418 (2002); Eur. Phys. J. A14, 469 (2002).

[11] T. Gross-Boelting, C. Fuchs, and A. Faessler, Nucl. Phys. A648, 105 (1999).

[12] S. L. Shapiro and S. A. Teukolsky, Black Holes, White Dwarfs, and Neutron Stars (John Wiley \& Sons, New York, 1983).

[13] J. Antoniadis et al., Science 340, 1233232 (2013). 